\title{
9 Politische Kontrafaktik
}

Dass die Kontrafaktik in der Gegenwart verstärkt als Verfahren politischen Schreibens genutzt wird, lässt sich mit Blick auf das literarische Feld der Gegenwart kaum ernsthaft bestreiten. Über die Gründe der Verbindung zwischen Kontrafaktik und politischem Schreiben ist damit freilich noch nichts ausgesagt. Will man verstehen, weshalb Kontrafaktik häufig politische Kontrafaktik ist - und zwar nicht nur in der Gegenwartsliteratur -, so kann man sich nicht allein auf empirisch-literarhistorische Befunde berufen, sondern wird sich den abstrakteren Voraussetzungen, Strukturen und Wirkungen kontrafaktischen Erzählens zuwenden müssen. Im Folgenden soll gezeigt werden, dass sowohl die basalen kognitiven Mechanismen des kontrafaktischen Denkens als auch die Referenzstruktur kontrafaktischer Werke die Kontrafaktik in besonderer Weise zum Verfahren politischen Schreibens prädestinieren. Kontrafaktik manifestiert sich sehr häufig in Form politischer Realitätsvariationen. Zur Erhärtung dieser These wird im Rahmen dieses Kapitels auf Erkenntnisse der Fiktionstheorie - inklusive des fiktionstheoretischen Teils der vorliegenden Arbeit - zurückgegriffen, darüber hinaus aber auch auf Forschungsbefunde der Psychologie und der Geschichtswissenschaft.

Die primären Funktionen kontrafaktischen Denkens hat die bisherige Literaturwissenschaft im epistemischen Bereich verortet: Gefragt wurde nach dem Erkenntnispotenzial kontrafaktischer Werke, etwa ihrer (vermeintlichen) Fähigkeit zur Problematisierung von Annahmen über Freiheit und Determinismus im historischen Prozess oder ihrer Kommentarfunktion in Bezug auf die (Un-) Möglichkeit objektiven historischen Wissens. ${ }^{547}$ Der Zusammenhang von Kontrafaktik und politischem Schreiben hingegen wurde bisher in der Literaturwissenschaft nicht intensiver diskutiert. ${ }^{548}$ Zweifellos sind die Funktionen kontrafaktischen Denkens - sowohl in der Kunst als auch darüber hinaus - vielfältig, sodass eine einseitige funktionale Festlegung des kontrafaktischen Denkens

547 Zur Problematik dieser Funktionsbestimmungen siehe Kapitel 3.2.1. Epistemische Übergeneralisierungen, sowie Kapitel 10. Historisches Erzählen als Kontrafaktik.

548 Die Herausgeber des Sammelbandes Interventionen in die Zeit. Kontrafaktisches Erzählen und Erinnerungskultur weisen darauf hin, dass die „Untersuchung der kulturellen Funktionen von Was-wäre-wenn-Szenarien noch in den Anfängen [steckt]“ (Nicolosi / Obermayr / Weller: Kontrafaktische Interventionen in die Zeit und ihre erinnerungskulturelle Funktion. Einleitung, S. 1).

Ә Open Access. (C) 2022 Michael Navratil, publiziert von De Gruyter. (c) BY-NC-ND Dieses Werk ist lizenziert unter einer Creative Commons Namensnennung - Nicht-kommerziell - Keine Bearbeitung 4.0 International Lizenz. https://doi.org/10.1515/9783110763119-011 
auf das politische Schreiben unzulässig wäre. ${ }^{549}$ Lutz Danneberg bemerkt in diesem Sinne, dass es „für das vielgliedrige Phänomen der kontrafaktischen Imaginationen keine kognitiven Standardfunktionen [gibt], die sie gleichsam per se erfüllen." 550 Danneberg und Andrea Albrecht benennen allerdings eine Reihe konkreter Funktionen, die kontrafaktische Imaginationen - also kontrafaktische Szenarien in faktualen Kontexten - unter Umständen erfüllen können: „Depending on the context, their function can be critical, affirmative, explanatory, heuristic, illustrative, or pedagogical. Counterfactual imaginations can be used to solve problems, analyze notions, and facilitate conclusions. “551 Die von Danneberg und Albrecht zusammengestellte Liste lässt dabei - ungeachtet des Nachdrucks auf der Multifunktionalität kontrafaktischen Denkens - eine deutliche Tendenz in Richtung politischer oder zumindest normativer Funktionsbestimmungen erkennen: Die Zuordnung einer etwaigen ,kritischen', affirmativen“ oder ,pädagogischen' Funktion zum Bereich des Politischen dürfte leicht nachzuvollziehen sein. Auch im Rahmen fiktionaler Texte erfüllen kontrafaktische Elemente zweifellos unterschiedliche Funktionen. Die politisch-normative Funktion scheint dabei allerdings besonders ausgeprägt zu sein.

Die Eignung der Kontrafaktik als Verfahren politischen Schreibens lässt sich jenseits aller literarhistorischen oder hermeneutischen Einzelbefunde - bereits auf einer basalen, kognitiv-psychologischen Ebene plausibilisieren. Zwar ist das Verständnis des kontrafaktischen Denkens in der Psychologie - ebenso wie in jedem anderen Fach - bis zu einem gewissen Grade disziplinspezifisch. Gleichwohl ist das psychologische Konzept kontrafaktischen Denkens intuitiv vergleichsweise leicht nachvollziehbar und hat darüber hinaus historisch wichtige Impulse für die Erforschung des kontrafaktischen Denkens in anderen Disziplinen geliefert, etwa in der Ökonomie, der Geschichts- oder der Politikwissenschaft. ${ }^{552}$ Es erscheint entspre-

549 Nünning hält fest, dass „es keine festen Korrelationen zwischen bestimmten Erzählstrukturen und spezifischen rezeptionsästhetischen Wirkungen und Funktionen [gibt].“ (Nünning: Von historischer Fiktion zu historiographischer Metafiktion. Bd. 1, S. 248).

550 Danneberg: Überlegungen zu kontrafaktischen Imaginationen in argumentativen Kontexten und zu Beispielen ihrer Funktion in der Denkgeschichte, S. 79.

551 Albrecht / Danneberg: First Steps Toward an Explication of Counterfactual Imagination, S. 16.

552 Vgl. Birke / Butter / Köppe: Introduction: England Win, S. 3. Zum Einfluss der psychologischen Forschung auf den rezenten Boom in der Erforschung kontrafaktischen Denkens siehe auch Roland Wenzlhuemer: Editorial: Unpredictability, Contingency and Counterfactuals. In: Ders. (Hg.): Counterfactual Thinking as a Scientific Method. Special Issue: Historical Social Research 34/2 (2009), S. 9-15, hier S. 12. 
chend naheliegend, zum Zweck einer basalen Funktionsbestimmung kontrafaktischen Denkens auf die einschlägige psychologische Forschung zu rekurrieren.

Kontrafaktisches Denken wird in der Psychologie seit den 1970er und verstärkt seit den 1990er Jahren erforscht. Mittlerweile liegen mehrere hundert Arbeiten zum Thema vor. In einem Überblicksartikel zur einschlägigen Forschung identifizieren Neal J. Roese und Mike Morrison zwei zentrale Mechanismen, welche den psychologischen Auswirkungen kontrafaktischen Denkens zugrunde liegen: nämlich den sogenannten causal inference effect und den contrast effect. ${ }^{553}$ Der causal inference effect bezeichnet die Fähigkeit kontrafaktischen Denkens, kausale Faktoren für eine bestimmte Entwicklung zu identifizieren, dadurch nämlich, dass diese Faktoren im Rahmen einer kontrafaktischen Imagination abgewandelt und die eingetretene Entwicklung damit hypothetisch verändert wird. Kontrafaktische Konditionale wie „Wenn ich früher losgegangen wäre, hätte ich den Bus nicht verpasst" lassen den basalen Mechanismus des causal inference effect deutlich werden. Häufig steht der causal inference effect im Zusammenhang mit einer Zuweisung von Verantwortlichkeit oder Schuld („,blaming effect“" ${ }^{554}$; er weist also eine deutliche Tendenz zu normativ-affektiven Bewertungen auf.

Den contrast effect wiederum charakterisieren Roese und Morrison wie folgt:

Counterfactual thoughts may influence emotions and judgments by way of a contrast effect, which is based on the juxtaposition of reality versus what might have been. [...] Contrast effects occur when a judgment is made more extreme via the juxtaposition of some anchor or standard [...]. For example, ice cream feels especially cold immediately after sipping hot tea. [...] Contrast effects also apply to subjective appraisals of value, satisfaction, and pleasure. Thus, by the same token, a factual outcome may be judged to be worse if a more desirable alternative outcome is salient, and that same outcome may be judged to be better if a less desirable alternative outcome is salient. ${ }^{555}$

Die normative Bewertung kontrafaktischer Szenarien kann dabei sowohl negativ als auch positiv ausfallen. Negativ bewertete Alternativen zur Realität werden als "downward counterfactuals“, positive als „upward counterfactuals“ bezeichnet. ${ }^{556}$

553 Vgl. Roese / Morrison: The Psychology of Counterfactual Thinking, S. 18-20.

554 Vgl. Roese / Morrison: The Psychology of Counterfactual Thinking, S. 18, 20. Hier trifft sich die psychologische Forschung mit der These Alexander Demandts, dass das Nachdenken über ungeschehene Geschichte in der Geschichtswissenschaft „zur Gewichtung von Kausalfaktoren“ sowie „zur Begründung von Werturteilen“ notwendig ist. Vgl. Demandt: Ungeschehene Geschichte, S. 3, 18-25.

555 Roese / Morrison: The Psychology of Counterfactual Thinking, S. 19.

556 Roese / Morrison: The Psychology of Counterfactual Thinking, S. 17. 
Die beschriebenen Erkenntnisse der Psychologie beziehen sich vorderhand auf allgemeine psychologische Mechanismen des Alltagsdenkens. Diese psychologischen Mechanismen lassen sich allerdings auch zur Erläuterung der Funktionen kontrafaktischer Kunstwerke heranziehen. So ist das Auslösen von Emotionen im Zusammenhang mit dem kontrafaktischen contrast effect zweifellos einer der Gründe, weswegen Leser oder Zuschauer sich überhaupt mit Werken der Kontrafaktik befassen: Über den Kontrasteffekt kontrafaktischen Denkens werden mitunter starke Emotionen evoziert und somit das Interesse an Werken der Kontrafaktik geweckt oder aufrechterhalten. ${ }^{557}$ Die beschriebenen Effekte liefern darüber hinaus eine Erklärung dafür, weshalb Kontrafaktik häufig als Verfahren politischen Schreibens eingesetzt wird: Sowohl die Zuweisungen von Schuld und Verantwortlichkeiten im Rahmen des causal inference effect als auch die kontrastive Verstärkung von Emotionen und Werturteilen im Rahmen des contrast effect lassen sich plausibel mit dem Bereich politischen Schreibens in Verbindung bringen. Wenn etwa am Ende von Tarantinos Inglourious Basterds Hitler und Goebbels von Mitgliedern einer amerikanisch-jüdischen Guerilla-Truppe erschossen werden, so leitet sich die emotionale Wirkung dieser Szene nicht allein aus der immanenten Dramaturgie des Films ab, sondern beruht wesentlich auch auf dem contrast effect bei der Imagination einer Welt, in welcher der Zweite Weltkrieg bereits 1944 endet, sowie dem causal inference effect, der sich aus der Suggestion ergibt, die Fortführung des Weltkriegs habe vom Wirken einzelner historischer Personen abgehangen: Die Ermordung von Hitler und Goebbels innerhalb der fiktionalen Welt hat schließlich ein früheres Ende des Kriegs zu Folge. Eine bestimmte Überzeugung hinsichtlich der Realgeschichte tritt somit gerade anhand der kontrafaktisch-amimetischen Variation derselben zutage: Da Hitler und die Nazi-Eliten für den Zweiten Weltkrieg verantwortlich waren, wäre es entschieden wünschenswert gewesen, dass sie rechtzeitig einem Attentat zum Opfer gefallen wären.

Das emotionale Kalkül von Tarantinos kontrafaktischem Kriegsfilm, welches auf dem historischen upward counterfactual bei der Imagination einer Ermordung

557 Roese / Morrison: The Psychology of Counterfactual Thinking, S. 21f. Die emotionale Wirkung der Kontrafaktik führen Roese und Morrison unter anderem auf dem Umstand zurück, dass das kontrafaktische Denken in der Kunst sich an Prozesse kontrafaktischen Vergleichens anlehnt, wie sie im menschlichen Gehirn ohnehin permanent ablaufen: „In an important sense, artists who use variations on a theme are mimicking the natural manner in which the human brain sees the world. [...] When the brain sees something surprising, the experience of surprise itself comes from the mental benchmarks that pop to mind and reveal how things could have (or should have) been. Brains are continuously producing creative variations (i. e., counterfactual elaborations of alternatives to current experiences) as we experience the flow of events in our lives.“ (ebd., S. 23). 
Hitlers beruht, hat im Übrigen ein nicht-fiktionales Komplement in der politischen Verehrung, die dem realen Grafen von Stauffenberg und den Mitorganisatoren des gescheiterten Hitler-Attentats vom 20. Juli 1944 entgegengebracht wird. ${ }^{558}$ Die zahlreichen fiktionalen Visionen einer Welt, in welcher die Nazis den Krieg gewonnen haben - etwa Robert Harris' Fatherland oder Philip K. Dicks The Man in the High Castle -, beruhen demgegenüber meist auf der emotionalen Kalkulation eines downward counterfactual, also einem kontrastiven Abgleich von fiktionaler und realer Welt, in dessen Rahmen die fiktionale Welt im Vergleich zu der realen Welt als schlechter bewertet wird.

Während sich die Literaturwissenschaft bisher kaum mit der politischen Funktion der Kontrafaktik auseinandergesetzt hat, wurde in der Geschichtswissenschaft die Tendenz des kontrafaktischen Erzählens zu normativen, affektiv eingefärbten oder politisch-tendenziösen Aussagen bereits vielfach diskutiert. Richard J. Evans etwa hält in seiner Überblicksdarstellung zum historischen kontrafaktischen Erzählen fest: „Kontrafaktische Darstellungen der Vergangenheit haben fast immer politische Implikationen für die Gegenwart. “559 In einem umfassenden Kapitel mit dem kritisch intendierten Titel „Wunschdenken“ ${ }^{\text {560 }}$ bietet Evans eine historisch weit ausgreifende Liste mit Beispielen kontrafaktischen Denkens, welche sehr deutlich normative, politische oder ideologische Interessen erkennen lassen. ${ }^{561}$ Mit seiner Problematisierung derartigen Wunschdenkens befindet sich Evans in Übereinstimmung mit der Mehrzahl der Historiker. Die Geschichtswissenschaft bewertet die, wenn man so will, politische Schlagseite des kontrafaktischen Denkens kritisch, da diese die angestrebte Objektivität historischer Erkenntnisse zu gefährden droht. Bereits Alexander Demandt (dem Evans übrigens seinerseits ebenfalls einen „Hang zum Wunschdenken“ unterstellt ${ }^{562}$ ) bemerkt in seinem Traktat Ungeschehene Geschichte:

Die meisten Alternativentwürfe wurzeln in Wünschen und Befürchtungen. Das Ungeschehene wird nicht einfach als anders, sondern als viel besser oder als viel schlechter darge-

558 Kontrafaktische Kalkulationen, die solchermaßen von den historischen Quellen selbst angeregt werden, dürften einen großen Einfluss auf die Bewertung von und das Interesse an bestimmten geschichtlichen Ereignissen ausüben. So könnte man etwa die These vertreten, das fortdauernde Interesse an der Kuba-Krise lasse sich letztlich auf die Faszination für die Möglichkeit eines - realiter nicht eingetretenen - Atomkriegs zurückzuführen.

559 Evans: Veränderte Vergangenheiten, S. 58.

560 Vgl. Evans: Veränderte Vergangenheiten, S. 17-58.

561 Bereits die ersten umfänglichen historisch-kontrafaktischen Texte aus dem mittleren 19. Jahrhundert lassen eine deutliche politische Stoßrichtung erkennen. Vgl. Evans: Veränderte Vergangenheiten, S. 21-27.

562 Evans: Veränderte Vergangenheiten, S. 46. 
stellt. Wie schön wäre es gewesen, wenn ... wie schlimm wäre es gewesen, wenn ... Der damit hervorgerufene Kontrast befriedigt unser Trostbedürfnis. Dieses mildert unsere Kritikfähigkeit und verfälscht die Plausibilitäten. ${ }^{563}$

Der Vorwurf lautet also, dass kontrafaktisches Denken auf der Basis historischen Materials kaum jemals neutrale kontrafaktische Alternativen gegen die Realität abwäge, sondern meist deutlich tendenziöse Alternativen entwerfe. Damit drohe allerdings die Strenge wissenschaftlichen Argumentierens verlorenzugehen: Anstatt auf der Basis realer Dokumente mögliche, wahrscheinliche oder plausible Alternativen des Geschichtsverlaufs zu entwerfen, verliere sich der Großteil alternativgeschichtlicher Szenarien - und zwar auch diejenigen aus der Feder von Historikern - in mehr oder minder realitätsenthobenen Spekulationen.

Auf eine politische Tendenz des kontrafaktischen Denkens innerhalb der Geschichtswissenschaft verweist nicht zuletzt der Umstand, dass kontrafaktische Szenarien vorwiegend von eher konservativen Historikern entwickelt werden. Kontrafaktische Szenarien in der Geschichtswissenschaft beschäftigen sich ,fast ausschließlich mit traditioneller, altmodischer Politik-, Militär- und Diplomatiegeschichte von der Art, wie sie in den 1950er Jahren vorherrschte. “564 Die reale Komplexität historischer Determinationsprozesse, welcher neuere Geschichtstheorien Rechnung zu tragen versuchen, wird somit in der Alternativgeschichtsschreibung kaum angemessen berücksichtigt. ${ }^{565}$ Kontrafaktische Szenarien, die

563 Demandt: Ungeschehene Geschichte, S. 95.

564 Evans: Veränderte Vergangenheiten, S. 171.

565 Mit ihrer konservativen Tendenz steht die kontrafaktische Geschichtsschreibung (Alternativgeschichte) damit in genauem ideologischen Gegensatz zur alternativen Geschichtsschreibung (Alternative History), welche historische Fakten nicht umschreibt, sondern diese Fakten vielmehr in ein gewandeltes Licht rückt oder neue historische Fakten zutage fördert, um damit einer bisher vernachlässigten Perspektive zu ihrem Recht zu verhelfen. Zur Alternative History siehe Kapitel 2.1. Terminologie, sowie Kapitel 10. Historisches Erzählen als Kontrafaktik. Die Gründe für diese unterschiedlichen politischen Tendenzen der beiden Formen der Geschichtsdarstellung sind vielfältig: Während die kontrafaktische Geschichtsschreibung notwendigerweise mit allgemein bekannten historischen Daten operiert - etwa den Lebensläufen großer Männer (Napoleon, Hitler etc.), berühmten Schlachten (Waterloo) oder veränderten Kriegsverläufen (vor allem des Amerikanischen Bürgerkriegs und des Zweiten Weltkriegs) - und somit implizit ein bestehendes Geschichtsbild weiter konsolidiert, liegt das Anliegen der alternativen Geschichtsschreibung meist in einer Subversion allgemein akzeptierter Annahmen über die Geschichte beziehungsweise in einer Neuperspektivierung derselben. Des Weiteren dürften hier auf weltanschaulicher Ebene unterschiedliche Haltungen zu Fragen des Geschichtsdeterminismus eine Rolle spielen: Eher links orientierte Historiker neigen dazu, entsubjektivierte Strukturfaktoren - der wirtschaftliche Unterbau, die Regeln der Sagbarkeit, Rassismen oder die Vorstellung von Geschlechterrollen etc. - als Determinanten der historischen Entwicklung hervorzuheben. Die alternative Geschichtsschreibung ist prädestiniert dafür, just diese Fakto- 
aus einer linken Perspektive verfasste wären, gibt es in der Geschichtswissenschaft kaum. ${ }^{566}$

Die Tendenz des kontrafaktischen Denkens zur Parteilichkeit, zur faktenungebundenen Spekulation sowie die mit kontrafaktischen Szenarien häufig einhergehende Konsolidierung eines konservativen, akteurs- und ereigniszentrierten Geschichtsbildes erweisen sich für den wissenschaftlich-faktualen Diskurs der Historiografie zweifellos als problematisch. Begegnet wird diesen Problemen in der Geschichtswissenschaft mit strengen methodischen Auflagen für das kontrafaktische Denken, etwa den Forderungen nach strikter Einhaltung von Plausibilitätskriterien, Faktenbindung und Wertneutralität (wobei anzumerken ist, dass bei konsequenter Berücksichtigung dieser methodischen Auflagen selbst noch die Mehrzahl der von Historikern verfassten kontrafaktischen Szenarien aus dem wissenschaftlichen Diskurs ausscheiden müsste ${ }^{567}$ ).

Für den Bereich literarischen Schreibens stellen a priori freilich weder eine fehlende Faktenbindung, die Reduktion von historischer Plausibilität noch auch die politische Tendenz eines Textes ein Problem dar. Als fiktionaler Diskurs ist die Kontrafaktik in Literatur, Film und Comic nicht an die Beschränkungen eines faktual-wissenschaftlichen Diskurses - inklusive dessen (vermeintliche) Wertneutralität - gebunden. Zurecht bemerken Johannes Rhein, Julia Schumacher und Lea Wohl von Haselberg: „Während das Wunschdenken [...] im Rahmen der Geschichtsschreibung aus guten Gründen als problematisch eingestuft werden kann, gilt dies nicht gleichermaßen zwingend für Werke innerhalb des fiktionalen Diskursrahmens. / Da Fiktionen einen eigenen kommunikativen Raum des

ren zu betonen. Mit den radikalen Alternativen des kontrafaktischen Denkens hingegen ist der beschriebene Strukturdeterminismus kaum vereinbar. Diese Unterscheidung von eher konservativer, kontrafaktischer Alternativgeschichtsschreibung und eher progressiv-linker, alternativer Geschichtsschreibung kann allerdings nur für das kontrafaktische Denken innerhalb der Geschichtswissenschaft Gültigkeit beanspruchen. Im Bereich der literarischen Kontrafaktik liegt keine vergleichbare Verbindung zwischen kontrafaktischem und konservativem Denken vor, wie im Rahmen der folgenden Interpretationen noch deutlich werden soll.

566 Vgl. Evans: Veränderte Vergangenheiten, S. 62.

567 Genau dies ist die Position von Richard J. Evans. Am Ende seiner Studie hält er zur kontrafaktischen Perspektive in der Geschichtswissenschaft fest, dass sie „[u]nter ganz bestimmten, streng begrenzten Umständen und für streng begrenzte Zwecke [...] nützlich sein [kann]; betrachtet man jedoch die umfangreiche Literatur, die diesem Genre mittlerweile zuzurechnen ist, mit Hunderten gedruckter Fallstudien, so kann man eigentlich nur zu dem Schluss kommen, dass sie vor allem als eigenständiges Phänomen von Nutzen und Interesse ist, als Teil der modernen, zeitgenössischen intellektuellen und politischen Geschichte, der selbst ein interessantes Studienobjekt darstellt, zur ernsthaften Erforschung der Vergangenheit jedoch keinen nennenswerten Beitrag leistet.“ (Evans: Veränderte Vergangenheiten, S. 196f.). 
,Als ob“ etablieren, sind sie von den Anforderungen des faktualen Diskurses entbunden. “568 Entsprechend unzulässig ist es, Werke der Kontrafaktik an den Maßstäben einer historischen Kontrafaktizitätsforschung zu messen, wie es in der Forschung bedauerlicherweise immer wieder geschehen ist. ${ }^{569}$

Anstatt eine eher randständige Gruppe streng epistemisch orientierter, skrupulös auf Plausibilität bedachter kontrafaktischer Geschichtsdarstellungen dogmatisch zum einzig legitimen Standard kontrafaktischen Erzählens zu erheben (was letztlich auf die tendenzielle Abschaffung des Erzählverfahrens hinausliefe), sollte man zunächst danach fragen, welche spezifischen Eigenschaften die Mehrzahl kontrafaktischer Szenarien - und zwar sowohl in der Literatur als auch in der Geschichtswissenschaft - aufweisen und was diesen Szenarien ihre Attraktivität verleiht. ${ }^{570}$ Man wird dann - übrigens in Übereinstimmung mit der psychologischen Forschung zum kontrafaktischen Denken - eine gleichsam natürliche Neigung des kontrafaktischen Erzählens zu Emotionsevokation, Spekulation und Normativität und mithin auch zur Politisierung konzedieren müssen. Diese Neigung mag zwar mit den Interessen einer streng szientifisch orientierten Geschichtswissenschaft nur bedingt vereinbar sein. Mit den ästhetischen Interessen künstlerischer Diskurse hingegen trifft sie sich mitunter aufs Günstigste. Was der Wissenschaft hier Hemmnis ist, ist der Kunst Gelegenheit: Während die vonseiten der Geschichtswissenschaft häufig beobachtete normativ-affektive Tendenz des kontrafaktischen Erzählens für den eigenen Fachdiskurs ein Problem darstellt, eröffnet dieselbe normativ-affektive Tendenz für die Literatur produktive Möglichkeiten, um die Kontrafaktik als Verfahren politischen Schreibens zum Einsatz zu bringen. ${ }^{571}$ Unter

568 Rhein / Schumacher / Wohl von Haselberg: Einleitung, S. 28.

569 Siehe Kapitel 3.2.2. Anlehnung an die Geschichtswissenschaft.

570 Auch Doležel hält fest: „[I]t is often difficult to decide whether the counterfactual historian is engaged in cognitive thought experiment or in the composition of a fantastic story. The link [to fiction-making] is even more intimate, owing to the existence of counterfactual historical fiction, such as H. Robert Harris's Fatherland (1992) or Carlos Fuentes's Terra Nostra (1975).“ (Doležel: Fictional and Historical Narrative, S. 267).

571 Auch für Konrad bildet gerade die affektive Dimension kontrafaktischen Denkens eine Bedingung für die Produktivität der historischen Kontrafaktik: „Tatsächlich bestätigen psychologische Studien und Experimente, dass es eine Reihe von Gefühlen gibt, die auf kontrafaktische Gedankengänge geradezu angewiesen sind: Dazu gehört insbesondere das Bedauern, das kaum möglich ist ohne ein Wissen darum, was unter anderen Voraussetzungen hätte geschehen können. Deshalb kann auch das literarische Kontrafaktische ein entscheidendes Mittel zur Aufarbeitung des Vergangenen sein. Nicht vergessen werden darf zudem, dass die Auseinandersetzung mit dem Erinnerten und Verdrängten in dieser speziellen Form zum einen durch den Kontrast auf eine Bewertung und Erkenntnis der Gegenwart und zum anderen durch Analogie und Prognosen für die Zukunft abzielt.“ (Eva-Maria Konrad: Literarische Gegenwelten. 
Berücksichtigung der natürlichen normativen Tendenz kontrafaktischen Denkens erweist sich letztlich nicht die (faktuale) Kontrafaktizität der Geschichtswissenschaft, sondern die (fiktionale) Kontrafaktik in Literatur, Film und Comic als der ,Normalfall‘ kontrafaktischen Erzählens.

Die besondere Eignung der Kontrafaktik als Verfahren politischen Schreibens lässt sich schließlich auch im Rückgriff auf die fiktionstheoretischen Überlegungen der vorliegenden Arbeit plausibilisieren. ${ }^{572}$ Traditionell existieren nur wenige Überschneidungen zwischen dem Bereich der Fiktionstheorie und der Forschung zur politischen Literatur: Fiktionstheoretische Untersuchungen befassen sich nur selten mit politischen Fragestellungen, und die Mehrzahl der Untersuchungen zur politischen Literatur lässt den genaueren fiktionstheoretischen Status ihrer Forschungsgegenstände unreflektiert. ${ }^{573}$ Dieses weitgehende Fehlen einer Schnittmenge zwischen den genannten Forschungsfeldern kann aus ihrem differierenden primären Erkenntnisinteresse sowie ihren verschiedenen Investigationsmethoden erklärt werden: Während sich die Fiktionstheorie als ein Teilbereich der Literaturwissenschaft mit streng analytischem, systematischem oder deskriptivem Anspruch versteht und als solcher der Linguistik, der analytischen Philosophie und teils sogar den Kognitionswissenschaften nahesteht, operiert die Diskussion um ,politische Literatur' und ,politisches Schreiben' oftmals mit starken normativen und weltanschaulichen Vorannahmen; sie bewegt sich damit eher in der Nähe zur Ethik sowie zu bedeutenden Teilen der kontinentalen Philosophie. Im Sinne einer heuristischen, im Detail freilich differenzierungsbedürftigen Oppositionsbildung könnte man vom epistemischen Interesse der Fiktionstheorie im Gegensatz zum normativen Interesse der Forschungen zur politischen Literatur sprechen.

Bedauerlich ist die beschriebene Trennung der Forschungstraditionen unter anderem deshalb, weil fast alle Versuche einer Beschreibung fiktionaler politischer Literatur explizit oder implizit davon ausgehen, dass politische Texte sich in irgendeiner Weise auf die außerliterarische Realität beziehen: etwa indem sie auf diese einzuwirken versuchen, sie kommentieren, kritisieren, subvertieren

In: Heribert Tommek / Matteo Galli / Achim Geisenhanslüke (Hg.): Wendejahr 1995. Transformationen der deutschsprachigen Literatur. Berlin / Boston 2015, S. 218-234, hier S. 234).

572 Zur Kopplung von Realität und Fiktion im Kontext des politischen Schreibens siehe auch Michael Navratil: Einspruch ohne Abbildung. Zur doppelte Diskursivität von Kathrin Rögglas Dokumentarismus. In: Iuditha Balint / Tanja Nusser / Rolf Parr (Hg.): Kathrin Röggla. München 2017, S. 143-160, hier bes. S. 144-148; ders.: Jenseits des politischen Realismus.

573 Eine positive Ausnahme bildet die Studie von Johannes Franzen: Indiskrete Fiktionen. Theorie und Praxis des Schlüsselromans 1960-2015. Göttingen 2018. 
oder auch affirmieren. Diese Realitätsanbindung politischer Literaturbetrachtungen wirft jedoch unausweichlich fiktionstheoretische Fragestellungen auf, etwa diejenige nach der realweltlichen Referenzialisierbarkeit diegetischer Elemente, nach der Kontextrelativität von Textdeutungen oder allgemein nach den Bedingungen transfiktionaler Interpretationsaussagen. Zugespitzt formuliert: Die Fiktionstheorie kann für die Mehrzahl ihrer Fragestellung auf eine Berücksichtigung des Politischen verzichten; eine Theorie politischer Literatur jedoch, die fiktionstheoretische Fragen vollständig ausklammert, ist unvollständig.

Nun kann die Fiktionstheorie natürlich nicht auf sich allein gestellt die eingangs beschriebenen Probleme einer Bestimmung des ,Politischen“ in der Literatur lösen. Sehr wohl kann sie aber dazu beitragen, jene Operationen zu charakterisieren, die bei der Interpretation eines Textes als ,politisch' vorgenommen werden. Lässt man vorderhand alle historischen, semantischen und praxeologischen Feinbestimmungen dessen, was genau ,Literatur' und ,das Politische“ bedeuten, außen vor, so kann festgehalten werden, dass ,politische Literatur` zumindest die eine Bedingung wird erfüllen müssen, sich in irgendeiner Weise zur politischen oder gesellschaftlichen Realität zu verhalten: Politische Literatur, so könnte eine fiktionstheoretische Minimaldefinition lauten, liegt vor, wenn ein fiktionaler Text im Rahmen der Interpretation mit normativen Implikationen zur Realität in Beziehung gesetzt wird. Nikolaus Wegmann bemerkt entsprechend im Reallexikon der deutschen Literaturwissenschaft: „In einem naiv-klassifikatorischen Sinne bezeichnet Politische Dichtung jede Literatur, die sich ihrem Stoff nach auf politische Sachverhalte - z. B. Kolonialismus, Patriotismus, soziale Spannungen - bezieht. ${ }^{\text {574 }}$ Politische Literatur kann mithin niemals radikal autonom sein, sondern muss, eben um politisch zu sein, auf die außerliterarische Realität Bezug nehmen. „Eine Literatur, die die Verhältnisse treffen will“, so Wegmann zur ,kritischen Literatur“, „muß auf eben diese nicht-literarische Realität hin lesbar sein.“575 Im Gegensatz hierzu wird man eine streng werkimmanente Interpretation - sofern eine solche überhaupt denkbar ist - kaum als politische Interpretation ansehen können. Bezeichnenderweise traten die historischen Proponenten der immanenten Interpretation - Wolfgang Kayser, Emil Staiger u. a. - zugleich auch für eine Abkehr von der politischen Kunstbetrachtung ein. ${ }^{576}$ Die konkrete Ausprägung des

574 Nikolaus Wegmann: Politische Dichtung. In: Jan-Dirk Müller (Hg.): Reallexikon der deutschen Literaturwissenschaft. Bd. III. Berlin 2003, S. 120-123, hier S. 120.

575 Wegmann: Engagierte Literatur?, S. 358.

576 Der Schule der werkimmanenten Interpretation wurde häufig Ignoranz gegenüber den realweltlichen Kontexten eines Textes - insbesondere dem historischen Wissen - vorgeworfen. Zumindest in verabsolutierter Form dürfte dieser Vorwurf jedoch nicht aufrechtzuerhalten sein. Danneberg bemerkt hierzu: „Das Kontextwissen zu einem Text erhält bei der werkimmanenten In- 
Realitätsbezugs politischer Literatur kann freilich sehr unterschiedlich ausfallen: Die Möglichkeiten reichen von einer dezidierten Affirmation der Realität (etwa in der Propaganda oder Panegyrik) über eine Kritik derselben (in der engagierten oder subversiven Literatur) bis hin zu einer ostentativen Indifferenz gegenüber der politischen Realität, die mitunter selbst wiederum als politische Positionierung angesehen werden kann. ${ }^{577}$

Die politische Interpretation eines fiktionalen Textes läuft also in jedem Fall auf eine Form transfiktionaler Aussagen hinaus: Ein bestimmter Aspekt der fiktionalen Welt wird in wertender Weise mit der realen Welt in Beziehung gesetzt. ${ }^{578}$ Beispiele für eine solche Korrelierung von fiktionaler und realer Welt im Rahmen politischer Interpretationen sind mühelos bei der Hand: Eine politische Deutung von Brechts Dreigroschenoper könnte etwa die Situation der Bettler, Huren und Zuhälter innerhalb der fiktionalen Welt in Beziehung zur Situation sozial benachteiligter und/oder krimineller Personen in der realen Welt setzen. Eine politische Deutung von Aldous Huxleys Brave New World könnte eine Beziehung zwischen der kapitalistischen Dystopie des Romans und den realen kapitalistischen Verhältnissen seiner Entstehungs- oder Rezeptionszeit herstellen. Und selbst die politische Deutung eines vordergründig so ostentativ apolitischen Romans wie Adalbert Stifters Nachsommer könnte nach den textuellen Symptomen fragen, über die sich die Verdrängung der realen gesellschaftlichen Antagonismen des mittleren 19. Jahrhunderts im Text verrät.

\footnotetext{
terpretation keine uneingeschränkte Priorität. Doch im Unterschied zu einem Teil der Interpretationspraxis und zu einer in der kritischen Rezeption verbreiteten Auffassung legitimiert diese Sicht des Kontextes keineswegs historische Ignoranz. “ (Lutz Danneberg: Zur Theorie der werkimmanenten Interpretation. In: Wilfried Barner / Christoph König (Hg.): Zeitenwechsel. Germanistische Literaturwissenschaft vor und nach 1945. Frankfurt a. M. 1996, S. 313-342, hier S. 318).

577 Selbst Adornos dialektische Verquickung von Engagement und Autonomie in seinem Engagement-Essay läuft auf eine normativ aufgeladene Korrelierung von Kunst und Realität hinaus: „Noch im sublimiertesten Kunstwerk birgt sich ein Es soll anders sein [...]. Vermittelt aber ist das Moment des Wollens durch nichts anderes als durch die Gestalt des Werkes, dessen Kristallisation sich zum Gleichnis eines Anderen macht, das sein soll. Als rein gemachte, hergestellte, sind Kunstwerke, auch literarische, Anweisungen auf die Praxis, deren sie sich enthalten: die Herstellung richtigen Lebens.“ (Adorno: Engagement, S. 429).

578 Natürlich ist diese Bindung politischer Interpretationen an transfiktionale normative Aussagen im Detail differenzierungsbedürftig. Nicht abgedeckt wird dadurch die politische Interpretation von Texten, die nicht oder nicht eindeutig fiktional sind, etwa von Lyrik. Des Weiteren nicht abgedeckt werden vom vorgeschlagenen Modell politische Interpretationen, die sich allein auf extratextuelle Aspekte wie die Selbstinszenierung eines Autors beziehen. Allerdings handelt es sich in solchen Fällen ohnehin nicht mehr eigentlich um Interpretationen politischen Schreibens im Sinne politischer (fiktionaler) Literatur.
} 
Die Notwendigkeit einer Korrelierung von fiktionaler und realer Welt im Rahmen des Interpretationsprozesses verbindet das politische Schreiben nun gerade mit der Kontrafaktik. Kontrafaktische Elemente zeichnen sich, so wurde im Theorieteil der Arbeit ausgeführt, durch transfiktionale Doppelreferenzen aus, durch explizite Referenzen also auf Elemente einer fiktionalen Welt, welche dann vom Leser durch implizite Referenzen auf die reale Welt ergänzt werden müssen, sodass sich eine weltenverbindende Vergleichsstruktur ergibt. ${ }^{579}$ Diese notwendige Transfiktionalität der Kontrafaktik korrespondiert auf günstige Weise mit einer Grundeigenschaft der politischen Literatur, die sich ebenfalls zur realen Welt hin öffnen muss, um als politische Literatur rezipierbar $\mathrm{zu}$ sein. Hat man es mit einer kontrafaktischen Referenzstruktur - also einem Vergleich von realer und fiktionaler Welt - zu tun, so ist nur noch ein kleiner Schritt erforderlich, um diesen transfiktionalen Weltvergleich zugleich normativ aufzuladen, sodass sich eine Form der politischen Realitätsvariation ergibt.

Ausgehend von dieser referenztheoretischen Strukturanalogie zwischen Kontrafaktik und politischer Literatur bieten sich für die Kontrafaktik vielfältige Möglichkeiten, um als Verfahren politischen Schreibens zum Einsatz zu kommen: Kontrafaktik kann politische Positionen und dominante Sprachformen mittels Überspitzung oder Umformung der Lächerlichkeit preisgeben (etwa in der politischen Satire oder in kreativen Formen des Dokumentarismus). Sie kann allgemein akzeptierte Geschichtsnarrative konterkarieren (in der Alternativgeschichte). Sie kann wünschenswerte Alternativen zum politischen Status quo aufzeigen (in der Utopie). Oder sie kann auf die Gefahren aktueller gesellschaftlicher oder technischer Entwicklungen und politischer Trends hinweisen (etwa in der Science-Fiction oder in der Dystopie). Angesichts dieser vielfältigen Einsatzmöglichkeiten überrascht es nicht, dass die Kontrafaktik gerade in der Gegenwartsliteratur, mit ihrer generellen Konjunktur amimetischer Erzählformen, zu einem bedeutenden Verfahren politischen Schreibens avancieren konnte.

Verstärkt wird diese Bindung von Kontrafaktik und politischem Schreiben durch den Umstand, dass die in kontrafaktischen Werken variierten realweltlichen Fakten oftmals bereits an und für sich eine hohe politische Brisanz aufweisen. Diese politische Aufladung des Faktenmaterials der Kontrafaktik ist dabei keineswegs zufällig, sondern hängt mit den kommunikationspragmatischen Erfordernissen des kontrafaktischen Erzählens zusammen: Für eine erfolgreiche Aktualisierung kontrafaktischer Referenzstrukturen ist es von Vorteil, wenn die in Frage stehenden Fakten als allgemein bekannt vorausgesetzt werden können; eine Abweichung von bloß klandestinem Wissen liefe Gefahr, unerkannt zu

579 Siehe die Kapitel 4.3.5. Transfiktionale Doppelreferenz. 
bleiben. Für die Aktualisierung kontrafaktischer Referenzstrukturen günstig ist es darüber hinaus, wenn dem relevanten Faktenmaterial eine hohe affektive, normative, politische oder anderweitige Bedeutung zukommt. Wird spezifischen Fakten besondere Relevanz beigemessen, so darf davon ausgegangen werden, dass ihre Variation innerhalb eines fiktionalen Mediums auch erkannt und für deutungsrelevant befunden wird. Es kann mithin kaum überraschen, dass gerade politisch brisante Themen das bevorzugte Ausgangsmaterial kontrafaktischer Faktenvariationen bilden; Bekanntheit und (affektive) Relevanzzuschreibung treten hier in ein Verhältnis der gegenseitigen Abhängigkeit und Verstärkung. Das Wissen etwa über den Ausgang des Zweiten Weltkriegs ist nicht nur den allermeisten Lesern bekannt, sondern darüber hinaus auch mit starken politischen, normativen oder affektiven Wertungen verbunden. Es kann somit einigermaßen zuverlässig damit gerechnet werden, dass die kontrafaktische Variation dieses Kriegsausgangs im Rahmen eines kontrafaktischen Romans wie etwa Robert Harris' Fatherland vom Leser nicht nur erkannt, sondern auch als intellektuell anregend, politisch-inkorrekt, provokativ, gewagt, absurd oder anderweitig interessant eingeschätzt - und genau in dieser spezifischen Qualität bei einer Deutung des Textes berücksichtigt werden wird. Kontrafaktische Texte werden dabei gleichsam mitaffiziert von der politischen Valenz, die den von ihnen variierten Themen und Fakten bereits realweltlich zukommen. ,Parasitär‘ verhalten sich Werke der Kontrafaktik also nicht nur in Bezug auf die epistemischen Eigenschaften des von ihnen variierten realweltlichen Faktenmaterials, sondern auch auf dessen normative oder affektive Aufladung.

Ausgehend von diesen fiktionstheoretischen Betrachtungen kann abschließend noch einmal zum Vorwurf des Eskapismus zurückgekehrt werden, der gegen die amimetische und insbesondere gegen die fantastische Literatur verschiedentlich erhoben wurde. Weiter oben wurde vorgeschlagen, das Politische in der Literatur fiktionstheoretisch über eine normative Beziehung zwischen Literatur und Welt zu definieren. Im Rahmen der Überlegungen zu möglichen real-fiktionalen Weltvergleichsverhältnissen - also Realistik, Fantastik, Kontrafaktik und Faktik - wurde ferner darauf hingewiesen, dass sich Elemente innerhalb fiktionaler Welten grundsätzlich mit der realen Welt vergleichen lassen, wobei zwischen Abweichung und Nicht-Abweichung sowie zwischen konkreten Referenzen und einem Bezug auf allgemeine Realitätsannahmen unterschieden werden kann. Entsprechend wäre es unzutreffend zu behaupten, dass fantastische Elemente gar keine Verbindung zur realen Welt unterhalten. Nur beruht diese Verbindung eben auf einer Abweichung von allgemeinen Realitätsannahmen, etwa der Nicht-Existenz von Drachen oder Feen. Fantastische Texte weichen also von der Realität ab, ohne dabei spezifische Realitätsreferenzen zu produzieren. Dies dürfte einer der zentralen Gründe dafür sein, dass die fantas- 
tische Kunst sich häufig dem Vorwurf des politischen Eskapismus ausgesetzt gesehen hat: Fiktionale Welten, die keine Verbindung zu Konkreta der realen Welt aufweisen, bieten sich für politische Deutungen nur eingeschränkt an.

Nun wurde in der Forschung wiederholt der Versuch unternommen, auch der fantastischen Kunst eine kritische oder politische Funktion zuzuschreiben. Rosemary Jackson etwa begreift - im Rückgriff auf Kategorien der Lacan'schen Psychoanalyse - das Fantastische als einen Gegendiskurs, welcher die dominante Ordnung des Symbolischen kritisch zu reflektieren und zu subvertieren vermag. ${ }^{580}$ Jackson reagiert damit auf einen prominenten ideologiekritischen Vorwurf, der von Forschern wie etwa Lars Gustafsson gegen die Fantastik erhoben wurde: dass nämlich die Fantastik ,ein gefährliches, ein menschlich bedrohliches Milieu“ sei, welches in einem antiaufklärerischen Affekt die Erkenntnismöglichkeiten der Vernunft in Frage stelle. ${ }^{581}$ Ähnlich wie Jackson wendet auch Hans Richard Brittnacher die Affinität des Fantastischen zum Hässlichen, Schrecklichen und rational Uneinholbaren funktional ins Positive, indem er die kanonkritische und kulturanthropologische Relevanz der Fantastik betont: „Die Nähe der Phantastik zu Blut, Sex und Tod“, so Brittnacher, „zeigt nicht nur ihr Mißtrauen gegen die sublimierende Deutungskultur der bürgerlichen Gesellschaft, sondern auch ihre Sympathie mit dem an der Moderne verzweifelnden Menschen. Seinen Ängsten, die in der bildungsbürgerlichen Ästhetik nicht einmal zur Darstellung gelangen, verhilft sie mit eigentümlich tröstenden archaischen Bildern zum Ausdruck. “582 Für Markus May wiederum bildet die Fantastik ein Medium der Erkenntniskritik, indem sie ,als Archiv des kulturell und epistemologisch Ver-

580 Jackson schreibt: „From a rational, 'monological' world, otherness cannot be known or represented except as foreign, irrational, 'mad', 'bad'. [...]. An understanding of the subversive function of fantastic literature emerges from structuralist rather than from merely thematic readings of texts. It has been seen that many fantasies from the late eighteenth century onwards attempt to undermine dominant philosophical and epistemological orders. They subvert and interrogate nominal unities of time, space and character, as well as questioning the possibility, or honesty, of fictional re-presentation of those unities. [...] [T] he fantastic can be seen as an art of estrangement, resisting closure, opening structures which categorize experience in the name of a 'human reality'. By drawing attention to the relative nature of these categories the fantastic moves towards a dismantling of the 'real', most particularly of the concept of 'character' and its ideological assumptions, mocking and parodying a blind faith in psychological coherence and in the value of sublimation as a 'civilizing' activity." (Rosemary Jackson: Fantasy: The Literature of Subversion. London / New York 1981, S. 173, 175f.).

581 Lars Gustafsson: Über das Phantastische in der Literatur. Ein Orientierungsversuch. In: Ders.: Utopien. München 1970, S. 9-25, hier S. 24. Vgl. Brittnacher / May: Phantastik-Theorien, S. 191.

582 Hans Richard Brittnacher: Ästhetik des Horrors. Gespenster, Vampire, Monster, Teufel und künstliche Menschen in der phantastischen Literatur. Frankfurt a. M. 1994, S. 7 f. 
drängten [fungiert]. Sie setzt sich zur Wehr gegen einen hegemonialen Diskurs von Modernität, dessen fundamentales Anliegen mit Max Webers berühmtem Diktum in der ,Entzauberung der Welt‘ angesiedelt werden muss. “583 Auch für Renate Lachmann wird in der Fantastik die „Begegnung der Kultur mit ihrem Vergessen“ inszeniert; die Leistung der fantastischen Literatur besteht Lachmann zufolge darin, dass sie „etwas in die Kultur zurückholt und manifest macht, was den Ausgrenzungen zum Opfer gefallen ist. Sie nimmt sich dessen an, was eine gegebene Kultur von dem abgrenzt, was sie als Gegenkultur oder Unkultur betrachtet. “584 Dorothee Girndt-Danneberg schließlich betont mit Blick speziell auf die fantastische Jugendliteratur, dass „,[d]istanzierende Darstellung realer und gemeinhin ,betreffender' Sachverhalte, Anleitung zu experimenteller Betrachtung des Gewohnten unter anderen Bedingungen, Demonstration folgerichtigen Schließens aus gegebenen Prämissen [...] notwendige Voraussetzungen kritischen Urteilens und Denken [seien]. Das Anknüpfen an kindlichen Vorstellungen aber erlaubt gerade, diese Vorstellungen zu präzisieren, zu erweitern oder zu korrigieren.“ ${ }^{585}$ Der Fantastik schreibt Girndt-Danneberg somit letztlich eine kognitiv förderliche Funktion zu.

Eine mehr oder weniger deutlich ausgeprägte politische Relevanzprätention ist allen diesen Deutungsversuchen der Fantastik zu eigen. Eine Verbindung zu politischen Konkreta der realen Welt wird in den angeführten Fantastik-Theorien allerdings kaum diskutiert. Die kritisch-politischen Potenziale der Fantastik werden hier eher auf den literarischen Kanon, auf das allgemeine Weltbild einer Kultur oder auf bestimmte kognitive Vorgänge bezogen, welche die Rezeption fantastischer Kunst ermöglicht - nicht aber auf spezifische Fakten der realen Welt.

Im Falle der Kontrafaktik liegen die Dinge jedoch anders: Im Gegensatz zur Fantastik bietet die Kontrafaktik die Möglichkeit, einerseits amimetisch zu erzählen, andererseits aber eine klare Verbindung zu Konkreta der realen Welt eben dem variierten, realweltlichen Faktenmaterial - aufrechtzuerhalten. Diese Bindung an Konkreta der realen Welt prädestiniert die Kontrafaktik in sehr viel höherem Maße als die Fantastik dazu, als Verfahren politischen Schreibens

583 Markus May: Die Wiederkehr der Dinge. Phantastik als Diskurskritik der Moderne. In: Marie-Thérèse Moury / Evelyne Jacquelin (Hg.): Phantastik und Gesellschaftskritik im deutschen, niederländischen und nordischen Kulturraum / Fantastique et approches critiques de la société. Espaces germanique, néerlandophone et nordique. Heidelberg 2018, S. 27-44, hier S. 28.

584 Lachmann: Erzählte Phantastik, S. 9, 11.

585 Dorothee Girndt-Dannenberg: Zur Funktion fantastischer Elemente in der erzählenden Jugendliteratur. In: Ernst Gottlieb von Bernstorff (Hg.): Aspekte der erzählenden Jugendliteratur. Eine Textsammlung für Studenten und Lehrer. Baltmannsweiler 1977, S. 149-185, hier S. 184. 
produktiv zu werden, indem sie nämlich qua Faktenvariation ebendiese Fakten kritisch kommentiert.

Die Opposition von eher politik-averser Fantastik und politik-affiner Kontrafaktik ist für eine Bewertung der politischen Potenziale verschiedener amimetischer Genres von großem Interesse. Der vorgeschlagenen Unterscheidung folgend könnte man nämlich die These vertreten, dass tendenziell fantastische Genres umso politischer werden, je stärker sie sich mit kontrafaktischen Referenzen ,aufladen'. So sind für die Genres der Science-Fiction oder der Fantasy kontrafaktische Referenzen fakultativ (anders als für die Alternativgeschichte, die per definitionem kontrafaktisch ist). Politische Lesarten von Science-Fiction und Fantasy sind nun aber häufig genau solche, in denen das fantastische Weltvergleichsverhältnis der jeweiligen Texte zusätzlich mit kontrafaktischen Referenzen unterlegt wird, in denen also realitätsdeviante Elemente nicht einfach als fantastische Elemente, sondern als Variationen realweltlichen Faktenmaterials betrachtet werden. Besonders deutlich wird diese Abhängigkeit der politischen Dimension amimetischer Texte von ihren kontrafaktischen Referenzen im Falle der Dystopie, bei der es sich qua Genre um eine Form politischen Schreibens handelt. Einen politischen Anspruch erheben dystopische Texte für gewöhnlich dadurch, dass sie bestimmte gesellschaftliche oder politische Tendenzen ihrer Entstehungszeit in die Zukunft projizieren und dabei deren negative Aspekte durch Übertreibung besonders augenfällig werden lassen. Dabei ist im Falle fiktionaler Dystopien der gegenwartskritische Aspekt für gewöhnlich bedeutender als der prognostische. Auch bei Dystopien ist es also gerade die (kontrafaktische) Bindung an reale Themen und Probleme der Gegenwart, die eine politische Lesart ermöglicht. Geht dystopische Literatur hingegen ihrer impliziten Verbindungen mit den politischen Problemen der Gegenwart verlustig, so büßt sie zugleich auch ihren Status als dystopische Literatur ein. Sie kippt dann gleichsam in den Genrebereich der Science-Fiction oder Fantasy. ${ }^{586}$

Abschließend kann festgehalten werden: Das politische Potenzial der Kontrafaktik rührt einerseits von den basalen psychologischen Funktionen her, die dem kontrafaktischen Denken immer und überall zukommen. Andererseits - und dies ist für die Literaturwissenschaft der bedeutendere, weil textanalytisch und interpretationspraktisch anschlussfähige Befund - bildet die Kontrafaktik die

586 So wird man etwa die Filme der Star Wars-Saga wohl kaum als Dystopien, sondern eher als Beispiele einer fantastischen Science-Fiction ansehen. Zwar werden hier durchaus politische Themen - Krieg, Wiederstand, politische Verfolgung etc. - verhandelt; doch unterhalten diese Themen in der Art ihrer filmischen Darstellung keine klare Verbindung zu konkreten Fakten der realen Welt. Eine politische Deutung der Star Wars-Filme, die ja Voraussetzung für eine Designation derselben als Dystopien wäre, erscheint mithin wenig plausibel. 
dialektisch intrikate Option eines Erzählverfahrens, das sich gleichzeitig von der Realität entfernt und an die Fakten der Realität bindet. Damit legt sie bereits qua narrativem Verfahren Deutungen nahe, welche einerseits die Relevanz der variierten Fakten für das kontrafaktische Werk herausstellen, andererseits aber auch die variierten Fakten selbst (kritisch) kommentieren. Ingo Irsigler bemerkt in diesem Zusammenhang zur historischen Kontrafaktik:

Weil diese fiktionale Kausalität von der Normalgeschichte abweicht, provoziert sie einerseits die Frage nach der Funktion dieser Abweichung mit Blick auf den tatsächlichen Geschichtsverlauf und befördert auf diese Weise eine historisch-politische Lesart des Textes. Andererseits betont sie aber auch die Eigenlogik der literarischen Fiktion gegenüber den Fakten. Der Gattung eignet demnach eine grundsätzliche Spannung zwischen Realitätsbezug und ästhetischer Realitätsnegation [.....$^{587}$

Der Befund, den Irsigler hier für den alternativgeschichtlichen Roman zieht, lässt sich in vergleichbarer Weise auch für andere kontrafaktische Genres wie Dystopie, Satire, Schlüsselroman oder Dokufiktion treffen: Gerade in der Realitätsvariation - der Begriff ,Variation‘ deutet es bereits an - bleibt die Kontrafaktik an die Realität gebunden. Bereits referenzstrukturell stellt die Kontrafaktik damit günstige Bedingungen für eine kritische oder politische Kommentierung ebendieser Realität bereit. Den Vorwurf der Realitätsflucht, welcher im Verhältnis zur fantastischen Kunst mitunter gerechtfertigt sein mag, wird man gegen die Kontraaktik nicht plausibel erheben können. ${ }^{588}$ Im Gegenteil erweist sind innerhalb der amimetischen Kunst gerade die Kontrafaktik als Königsweg zum Politischen.

587 Ingo Irsigler: World Gone Wrong. Christian Krachts alternativhistorische Antiutopie Ich werde hier sein im Sonnenschein und im Schatten. In: Hans-Edwin Friedrich (Hg.): Der historische Roman. Erkundung einer populären Gattung. Frankfurt a. M. 2013, S. 171-186, hier S. 174. 588 Zwar wurde auch gegen die kontrafaktische Kunst gelegentlich der Vorwurf des Eskapismus und, damit verbunden, der problematischen Komplexitätsreduktion erhoben. So bringt etwa Richard J. Evans seine Abneigung gegenüber allzu spekulativen Formen kontrafaktischen Erzählens dadurch zum Ausdruck, dass er gegen den Eskapismus von Werken wie Lord of the Rings oder Game of Thrones polemisiert. Vgl. Evans: Veränderte Vergangenheiten, S. 57, 196. Für eine Diskussion der Funktionen genuin kontrafaktischer Kunst sind die genannten Werke allerdings denkbar schlecht geeignet, stammen sie doch beide aus dem Genrebereich der Fantasy: Während die fantastischen Erzählwelten von Lord of the Rings oder Game of Thrones keine oder kaum konkrete Referenzen auf die reale Welt etablieren, unterhalten die kontrafaktischen Erzählwelten von Harris' Fatherland, Orwells 1984 oder Ransmayrs Morbus Kitahara eine enge Beziehung zur realen Welt, deren Fakten sie kontrafaktisch variieren. 